\title{
Synchronization of $N$ Coupled Chaotic Systems with Ring Connection Based on Special Antisymmetric Structure
}

\author{
Xiangyong Chen, ${ }^{1}$ Jianlong Qiu, ${ }^{1}$ Qiang Song, ${ }^{2}$ and Ancai Zhang ${ }^{3}$ \\ ${ }^{1}$ School of Sciences, Linyi University, Linyi 276005, China \\ ${ }^{2}$ School of Electronics and Information, Hangzhou Dianzi University, Hangzhou 310018, China \\ ${ }^{3}$ School of Automobile Engineering, Linyi University, Linyi 276005, China
}

Correspondence should be addressed to Jianlong Qiu; qiujianlong@lyu.edu.cn

Received 15 June 2013; Accepted 20 August 2013

Academic Editor: Xinsong Yang

Copyright ( 2013 Xiangyong Chen et al. This is an open access article distributed under the Creative Commons Attribution License, which permits unrestricted use, distribution, and reproduction in any medium, provided the original work is properly cited.

\begin{abstract}
This paper considers the complete synchronization problem for $N$ coupled chaotic systems with ring connections. First, we use a direct design method to design a synchronization controller. It transforms the error system into a stable system with special antisymmetric structure. And then, we get some simple stability criteria of achieving the complete synchronization. These criteria are not only easily verified but also improve and generalize previous known results. Finally, numerical examples are provided to demonstrate the effectiveness of the theoretical analysis.
\end{abstract}

\section{Introduction}

Since the pioneering work of Pecora and Carroll [1], chaotic synchronization has been intensively investigated due to its potential applications in many fields [2-4]. Several types of synchronization phenomena have been reported, such as complete synchronization [5], phase synchronization [6], antisynchronization [7], projective synchronization [8,9], and generalized synchronization [10]. To solve the problem of chaotic synchronization, many approaches have been developed. That includes a sliding mode control method [11], an impulsive control method [11], an adaptive control method [7, 12], a pinning control method [13], and a sampled-data control method [14]. However, these control algorithms are just suitable to synchronize two identical or nonidentical chaotic systems.

Nowadays, the synchronization of multiple chaotic systems has attracted increasing attention. It has been widely used in secure communication area in order to reduce the synchronizing cost of multiple chaotic communication and make simultaneous multiparty communications possible. Therefore, the synchronization in multiple chaotic systems has more advantages and deserves to be deeply investigated comparing with the conventional chaotic synchronization.
Several types of synchronization in an array of chaotic systems have been investigated in the past few years, for example, the global synchronization in $[15,16]$, and the adaptive coupled synchronization in [17], the projective synchronization in [18]. In [19-21], the synchronization of $N$ chaotic systems of with ring and chain connection was investigated. In addition, Yang and Zhang [22] studied the synchronization of an array of identical chaotic systems and discussed its application for secure communication with noise perturbation.

However, it is notable that the realization of synchronization of $N$ coupled chaotic systems is much more difficult. So, it is necessary to find an easy method that realizes the synchronization of such multiple chaotic systems. Cai et al. $[7,8]$ investigated the generalized projective synchronization of two different chaotic systems based on a special antisymmetric structure. We studied the problem of synchronization of $N$ different chaotic systems by using a special antisymmetric structure [21]. Inspired by the above discussions, we further discuss the synchronization of $N$ coupled chaotic systems in this paper. First, a synchronization controller that is designed by using a direct design method transforms the error system into a stable system with special antisymmetric structure. And then, we derive some sufficient conditions in order to guarantee the asymptotical stabilization of the error system 
at the origin. It means that the synchronization of $N$ coupled chaotic systems is realized.

The paper is organized as follows. In Section 2, the synchronization of $N$ coupled chaotic systems with ring connection is theoretically analyzed. A stability theorem for such systems with special antisymmetric structure is given. In Section 3, the synchronization control schemes are applied to three identical and non-identical coupled chaotic systems. Simulation results demonstrate the effectiveness of proposed schemes. And finally some concluding remarks are given in Section 4.

\section{Synchronization of $N$ Chaotic Systems and Controllers Design}

Consider $N$ coupled chaotic systems with ring connections described by

$$
\begin{aligned}
\dot{x}_{1} & =A_{1} x_{1}+g_{1}\left(x_{1}\right)+D_{1}\left(x_{N}-x_{1}\right), \\
\dot{x}_{2} & =A_{2} x_{2}+g_{2}\left(x_{2}\right)+D_{2}\left(x_{1}-x_{2}\right), \\
& \vdots \\
\dot{x}_{N} & =A_{N} x_{N}+g_{n}\left(x_{N}\right)+D_{N}\left(x_{N-1}-x_{N}\right),
\end{aligned}
$$

where $x_{1}, x_{2}, \ldots, x_{N}$ are the state vectors of the chaotic systems; $g_{i}\left(x_{i}\right)(i=1, \ldots, N)$ is the continuous nonlinear function; $A_{1}, A_{2}, \ldots, A_{N}$ are constant matrices; $D_{i}=\operatorname{diag}\left(d_{1 j}\right.$, $\left.\ldots, d_{N j}\right)$ and $d_{i j} \geq 0$ are the diagonal matrices which represent the coupled parameters. If

$$
\begin{gathered}
A_{i} \neq A_{j}, \quad i=1, \ldots N, \quad j=1, \ldots N, \quad i \neq j, \\
g_{i}(\cdot) \neq g_{j}(\cdot), \quad i=1, \ldots N, j=1, \ldots N, \quad i \neq j,
\end{gathered}
$$

then the system (1) is an array of non-identical chaotic systems.

Now the above simple coupling form is applied to investigate the synchronization of $N$ chaotic systems. The systems are

$$
\begin{aligned}
\dot{x}_{1} & =A_{1} x_{1}+g_{1}\left(x_{1}\right)+D_{1}\left(x_{N}-x_{1}\right), \\
\dot{x}_{2} & =A_{2} x_{2}+g_{2}\left(x_{2}\right)+D_{2}\left(x_{1}-x_{2}\right)+u_{1}, \\
& \vdots \\
\dot{x}_{N} & =A_{N} x_{N}+g_{n}\left(x_{N}\right)+D_{N}\left(x_{N-1}-x_{N}\right)+u_{N-1} .
\end{aligned}
$$

Let the state error be $e_{i-1}=x_{i}-x_{i-1},(i=2, \ldots, N)$. It is not difficult to obtain the following dynamical error system:

$$
\begin{aligned}
\dot{e} & =\left[\begin{array}{ccccc}
A_{2}-D_{1}-D_{2} & -D_{1} & -D_{1} & \cdots & -D_{1} \\
D_{2} & A_{3}-D_{3} & 0 & \cdots & 0 \\
0 & D_{3} & A_{4}-D_{4} & \cdots & 0 \\
\vdots & \vdots & \vdots & \vdots & \vdots \\
0 & 0 & 0 & \cdots & A_{N}-D_{N}
\end{array}\right]\left[\begin{array}{c}
e_{1} \\
e_{2} \\
e_{3} \\
\vdots \\
e_{N-1}
\end{array}\right] \\
& +\left[\begin{array}{c}
\left(A_{2}-A_{1}\right) x_{1}+g_{2}\left(x_{2}\right)-g_{1}\left(x_{1}\right)+u_{1} \\
\left(A_{3}-A_{2}\right) x_{2}+g_{3}\left(x_{3}\right)-g_{2}\left(x_{2}\right)+u_{2}-u_{1} \\
\vdots \\
\left(A_{N}-A_{N-1}\right) x_{N-1}+g_{N}\left(x_{N}\right)-g_{N-1}\left(x_{N-1}\right)+u_{N-1}-u_{N-2}
\end{array}\right] .
\end{aligned}
$$

Our purpose is to design the controllers $u_{i}(i=1, \ldots, N-$ 1) such that the error system (4) is asymptotically stable at the origin. That is,

$$
\lim _{t \rightarrow \infty}\left\|e_{i-1}\right\|=\lim _{t \rightarrow \infty}\left\|x_{i}-x_{i-1}\right\|=0
$$

This implies that the synchronization of chaotic systems (3) is realized.

Here a direct design control method $[7,8,21]$ is used to achieve the objective. This method presents an easy procedure of selecting proper controllers in chaos synchronization. So we adopt this method to transform the error system into a stable system with a special antisymmetric structure. The main results are given below.

First, we choose the control input $u_{i}$ to eliminate all known items that cannot be shown in the form of the error system $e_{i}$. The controllers are given by

$$
\begin{aligned}
u_{1}= & v_{1}-\left(A_{2}-A_{1}\right) x_{1}-g_{2}\left(x_{2}\right)+g_{1}\left(x_{1}\right), \\
u_{2}= & v_{2}-\left(A_{3}-A_{2}\right) x_{2}-g_{3}\left(x_{3}\right)+g_{2}\left(x_{2}\right)+u_{1}, \\
& \vdots \\
u_{N-1}= & v_{N-1}-\left(A_{N}-A_{N-1}\right) x_{N-1} \\
& -g_{N}\left(x_{N}\right)+g_{N-1}\left(x_{N-1}\right)+u_{N-2},
\end{aligned}
$$

where $\left[\begin{array}{llll}v_{1} & v_{2} & \cdots & v_{N-1}\end{array}\right]^{T}=H\left[\begin{array}{llll}e_{1} & e_{2} & \cdots & e_{N-1}\end{array}\right]^{T}$ and $H$ is a coefficient matrix. So, the error systems (4) are rewritten by

$$
\dot{e}=L(e) e,
$$

where

$$
L(e)=\left[\begin{array}{ccccc}
A_{2}-D_{1}-D_{2} & -D_{1} & -D_{1} & \cdots & -D_{1} \\
D_{2} & A_{3}-D_{3} & 0 & \cdots & 0 \\
0 & D_{3} & A_{4}-D_{4} & \cdots & 0 \\
\vdots & \vdots & \vdots & \vdots & \vdots \\
0 & 0 & 0 & \cdots & A_{N}-D_{N}
\end{array}\right]+H .
$$

Theorem 1. Consider the systems (7) with the state dependent coefficient $L(e)=L_{1}(e)+L_{2}$. If $L_{1}(e)$ and $L_{2}$ satisfy the following assumptions:

$$
\begin{gathered}
L_{1}^{T}(e)=-L_{1}(e), \\
L_{2}=\operatorname{diag}\left(l_{1}, \ldots, l_{n}\right), \quad l_{i}<0,(i=1, \ldots, n),
\end{gathered}
$$

then the system (7) is asymptotically stable, which means that the complete synchronization of $N$ coupled chaotic systems (3) are achieved.

Proof. Choose a Lyapunov function to be

$$
V=\frac{1}{2} e^{T} e .
$$

The derivative of $V$ is

$$
\dot{V}=\frac{1}{2}\left(\dot{e}^{T} e+e^{T} \dot{e}\right)=\frac{1}{2} e^{T}\left(L(e)^{T}+L(e)\right) e .
$$


Since $L(e)=L_{1}(e)+L_{2}, L_{1}^{T}(e)=-L_{1}(e)$ and $L_{2}=$ $\operatorname{diag}\left(l_{1}, \ldots, l_{n}\right), l_{i}<0,(i=1, \ldots, n)$, it is easy to get

$$
\dot{V}=e^{T} L_{2} e<0 .
$$

From Lyapunov stability theory, we know that the equilibrium $e=0$ of the system (7) is global asymptotically stable. Then the complete synchronization of $N$ chaotic systems (3) is achieved.

Remark 2. The error dynamic system (4) is transformed into the systems $\dot{e}=L(e) e$ under the control law $u_{i}$, where $L(e)$ possesses the antisymmetric structure. Theorem 1 ensures that the error system (4) is asymptotically stable at the origin.

Remark 3. There are many possible choices for $H$ as long as it guarantees the error dynamic system (7) to be asymptotically stable at the origin. Without loss of generality, we define $H$ to be a state dependent coefficient matrix. As a result, the sufficient stability conditions of the systems (7) are given by transforming it into a stable system with a special antisymmetric structure.

Remark 4. The antisymmetric structures in Theorem 1 are the generalization of the tridiagonal structures. The error system constructed with the antisymmetric structure is more convenient than the one with tridiagonal structure when the original system has some zero elements at the tridiagonal position and nonzero elements at other positions.

Since the antisymmetric structure is related to the coefficient matrices and the states of the original system, the selecting of the coefficient matrices with antisymmetric structure is an important and difficult task. In the next section, we will demonstrate the proposed approaches for the special structure through numerical examples.

\section{Applications of Synchronization Control Schemes}

In this section, we use two simulation examples to illustrate the effectiveness of the proposed schemes. The synchronization is simulated for the non-identical and identical chaotic systems, respectively.

Case 1. When the drive system and response systems are identical chaotic systems, the drive system and response systems are all the Lorenz chaotic system. They are described as follows:

$$
\begin{aligned}
& \dot{x}_{11}=-10 x_{11}+10 x_{12}+d_{11}\left(x_{31}-x_{11}\right), \\
& \dot{x}_{12}=28 x_{11}-x_{12}-x_{11} x_{13}+d_{12}\left(x_{32}-x_{12}\right), \\
& \dot{x}_{13}=-\frac{8}{3} x_{13}+x_{11} x_{12}+d_{13}\left(x_{33}-x_{13}\right), \\
& \dot{x}_{21}=-10 x_{21}+10 x_{22}+d_{21}\left(x_{11}-x_{21}\right)+u_{11}, \\
& \dot{x}_{22}=28 x_{21}-x_{22}-x_{21} x_{23}+d_{22}\left(x_{12}-x_{22}\right)+u_{12},
\end{aligned}
$$

$$
\begin{aligned}
& \dot{x}_{23}=-\frac{8}{3} x_{23}+x_{21} x_{22}+d_{23}\left(x_{13}-x_{23}\right)+u_{13}, \\
& \dot{x}_{31}=-10 x_{31}+10 x_{32}+d_{31}\left(x_{21}-x_{31}\right)+u_{21}, \\
& \dot{x}_{32}=28 x_{31}-x_{32}-x_{31} x_{33}+d_{32}\left(x_{22}-x_{32}\right)+u_{22}, \\
& \dot{x}_{33}=-\frac{8}{3} x_{33}+x_{31} x_{32}+d_{33}\left(x_{23}-x_{33}\right)+u_{23},
\end{aligned}
$$

where

$$
\begin{gathered}
A_{1}=A_{2}=A_{3}=A=\left[\begin{array}{ccc}
-10 & 10 & 0 \\
28 & -1 & 0 \\
0 & 0 & -\frac{8}{3}
\end{array}\right], \\
g_{1}\left(x_{1}\right)=\left[\begin{array}{c}
0 \\
-x_{11} x_{13} \\
x_{11} x_{12}
\end{array}\right], \quad g_{2}\left(x_{2}\right)=\left[\begin{array}{c}
0 \\
-x_{21} x_{23} \\
x_{21} x_{22}
\end{array}\right], \\
g_{3}\left(x_{3}\right)=\left[\begin{array}{c}
0 \\
-x_{31} x_{33} \\
x_{31} x_{32}
\end{array}\right],
\end{gathered}
$$

and $D_{1}=\operatorname{diag}\left(d_{11}, d_{12}, d_{13}\right), D_{2}=\operatorname{diag}\left(d_{21}, d_{22}, d_{23}\right), D_{3}=$ $\operatorname{diag}\left(d_{31}, d_{32}, d_{33}\right)$ are the coupled matrices, and $u_{1}=\left[u_{11}\right.$, $\left.u_{12}, u_{13}\right]^{T}$ and $u_{2}=\left[u_{21}, u_{22}, u_{23}\right]^{T}$ are the control inputs.

Let the synchronization error state be $\dot{e}_{i-1}=\dot{x}_{i}-\dot{x}_{i-1}$, $(i=2,3)$. The error dynamical equations are

$$
\dot{e}=\left[\begin{array}{ll}
\Gamma_{1} & \Gamma_{2} \\
\Gamma_{3} & \Gamma_{4}
\end{array}\right] e+\left[\begin{array}{c}
u_{11} \\
-x_{21} x_{23}+x_{11} x_{13}+u_{12} \\
x_{21} x_{22}-x_{11} x_{12}+u_{13} \\
u_{21}-u_{11} \\
-x_{31} x_{33}+x_{21} x_{23}+u_{22}-u_{12} \\
x_{31} x_{32}-x_{21} x_{22}+u_{23}-u_{13}
\end{array}\right]
$$

where

$$
\begin{aligned}
& \Gamma_{1}=\left[\begin{array}{ccc}
-10-d_{11}-d_{21} & 10 & 0 \\
28 & -1-d_{12}-d_{22} & 0 \\
0 & 0 & -\frac{8}{3}-d_{13}-d_{23}
\end{array}\right], \\
& \Gamma_{2}=\left[\begin{array}{ccc}
-d_{11} & 0 & 0 \\
0 & -d_{12} & 0 \\
0 & 0 & -d_{13}
\end{array}\right], \quad \Gamma_{3}=\left[\begin{array}{ccc}
d_{21} & 0 & 0 \\
0 & d_{22} & 0 \\
0 & 0 & d_{23}
\end{array}\right], \\
& \Gamma_{4}=\left[\begin{array}{ccc}
-10-d_{31} & 10 & 0 \\
28 & -1-d_{32} & 0 \\
0 & 0 & -\frac{8}{3}-d_{33}
\end{array}\right] .
\end{aligned}
$$

We design the controllers $u_{1}$ and $u_{2}$ to be

$$
\begin{gathered}
u_{1}=v_{1}-g_{2}\left(x_{2}\right)+g_{1}\left(x_{1}\right), \\
u_{2}=v_{2}+v_{1}-g_{3}\left(x_{3}\right)+g_{1}\left(x_{1}\right),
\end{gathered}
$$


where

$$
\begin{gathered}
v_{1}=\left[\begin{array}{cccccc}
0 & 0 & 0 & 0 & 0 & 0 \\
-38 & 0 & 0 & 0 & 0 & 0 \\
0 & 0 & 0 & 0 & 0 & 0
\end{array}\right] e, \\
v_{2}=\left[\begin{array}{cccccc}
d_{11}-d_{21} & 0 & 0 & 0 & 0 & 0 \\
38 & d_{12}-d_{22} & 0 & -38 & 0 & 0 \\
0 & 0 & d_{13}-d_{23} & 0 & 0 & 0
\end{array}\right] e .
\end{gathered}
$$

The error systems (15) become

$$
\dot{e}=\left[\begin{array}{cc}
\Gamma_{1}^{*} & \Gamma_{2}^{*} \\
\Gamma_{3}^{*} & \Gamma_{4}^{*}
\end{array}\right] e,
$$

where

$$
\begin{aligned}
& \Gamma_{1}^{*}=\left[\begin{array}{ccc}
-10-d_{11}-d_{21} & 10 & 0 \\
-10 & -1-d_{12}-d_{22} & 0 \\
0 & 0 & -\frac{8}{3}-d_{13}-d_{23}
\end{array}\right], \\
& \Gamma_{2}^{*}=\left[\begin{array}{ccc}
-d_{11} & 0 & 0 \\
0 & -d_{12} & 0 \\
0 & 0 & -d_{13}
\end{array}\right], \quad \Gamma_{3}^{*}=\left[\begin{array}{ccc}
d_{11} & 0 & 0 \\
0 & d_{12} & 0 \\
0 & 0 & d_{13}
\end{array}\right], \\
& \Gamma_{4}^{*}=\left[\begin{array}{ccc}
-10-d_{31} & 10 & 0 \\
-10 & -1-d_{32} & 0 \\
0 & 0 & -\frac{8}{3}-d_{33}
\end{array}\right] .
\end{aligned}
$$

If the conditions

$$
\begin{aligned}
& -10-d_{11}-d_{21}<0, \quad-1-d_{12}-d_{22}<0, \\
& -\frac{8}{3}-d_{13}-d_{23}<0, \quad-10-d_{31}<0, \\
& -1-d_{32}<0, \quad-\frac{8}{3}-d_{33}<0
\end{aligned}
$$

are satisfied, then Theorem 1 tells us that the error systems (19) are asymptotically stable at the origin under the controllers $u_{1}$ and $u_{2}$. It means that the synchronization of (13) is realized.

Fourth order Runge-Kutta integration method is used to numerical simulation with time step size 0.001 . Let the initial conditions of the drive system and the response systems be $\left(x_{11}(0), x_{12}(0), x_{13}(0)\right)=(4,5,-3),\left(x_{21}(0), x_{22}(0), x_{23}(0)\right)=$ $(5,2,-5)$, and $\left(x_{31}(0), x_{32}(0), x_{33}(0)\right)=(11,15,10)$. respectively. And we choose that $d_{12}=d_{22}=d_{32}=d_{13}=d_{23}=$ $d_{33}=0$ and $d_{11}=d_{21}=d_{31}=1$. The state trajectories of the error systems and chaotic systems are shown in Figures 1, 2, 3 , and 4 , respectively. It is easy to see that the state variables and the error variables all tend towards to less than $2 \mathrm{~s}$ under the controllers. Simulation results demonstrate that the convergence rates are much faster than the earlier research results proposed in the literature [19, 21]; then the effectiveness of the synchronization control schemes is confirmed.

Case 2. When the drive system and response systems are non-identical chaotic systems, the Chen system, Lü system,

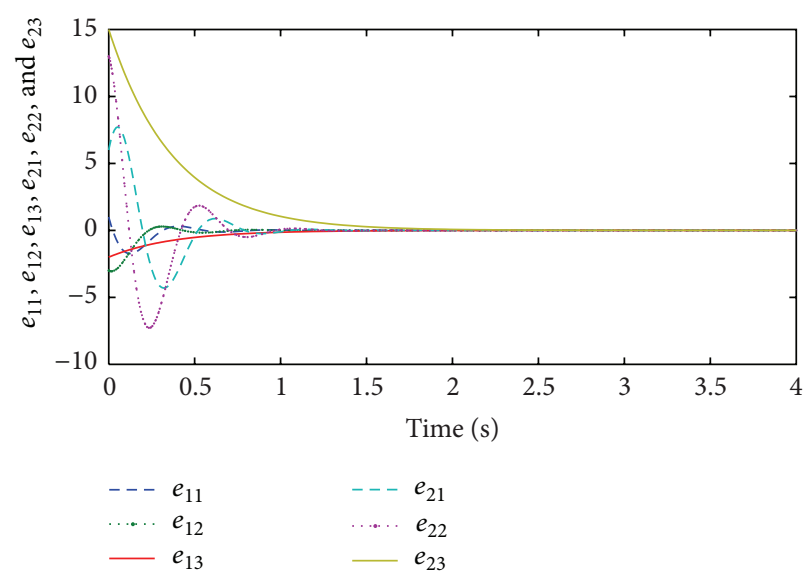

FIGURE 1: Dynamics of the variables $e_{11}, e_{12}, e_{13}, e_{21}$, and $e_{22}$ and $e_{23}$ of the error systems $e_{1}$ and $e_{2}$ with time $t$.

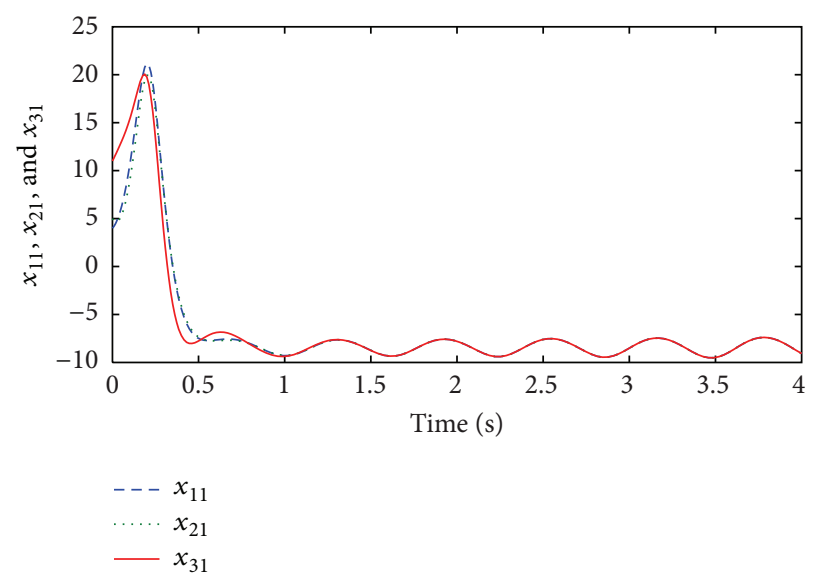

FIGURE 2: The state trajectories $x_{11}, x_{21}$, and $x_{31}$ of the Lorenz systems with time $t$.

and Lorenz system are considered as drive system and response systems, respectively. They are described as follows:

$$
\begin{aligned}
& \dot{x}_{11}=-35 x_{11}+35 x_{12}+d_{11}\left(x_{31}-x_{11}\right), \\
& \dot{x}_{12}=-7 x_{11}+28 x_{12}-x_{11} x_{13}+d_{12}\left(x_{32}-x_{12}\right), \\
& \dot{x}_{13}=-3 x_{13}+x_{11} x_{12}+d_{13}\left(x_{33}-x_{13}\right), \\
& \dot{x}_{21}=-36 x_{21}+36 x_{22}+d_{21}\left(x_{11}-x_{21}\right)+u_{11}, \\
& \dot{x}_{22}=20 x_{22}-x_{21} x_{23}+d_{22}\left(x_{12}-x_{22}\right)+u_{12}, \\
& \dot{x}_{23}=-3 x_{23}+x_{21} x_{22}+d_{23}\left(x_{13}-x_{23}\right)+u_{13}, \\
& \dot{x}_{31}=-10 x_{31}+10 x_{32}+d_{31}\left(x_{21}-x_{31}\right)+u_{21}, \\
& \dot{x}_{32}=28 x_{31}-x_{32}-x_{31} x_{33}+d_{32}\left(x_{22}-x_{32}\right)+u_{22}, \\
& \dot{x}_{33}=-\frac{8}{3} x_{33}+x_{31} x_{32}+d_{33}\left(x_{23}-x_{33}\right)+u_{23},
\end{aligned}
$$




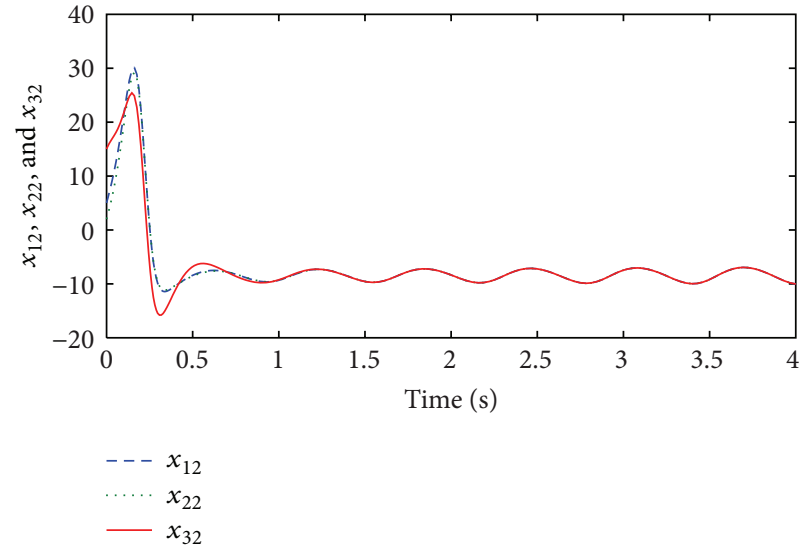

Figure 3: The state trajectories $x_{12}, x_{22}$, and $x_{32}$ of the Lorenz systems with time $t$.

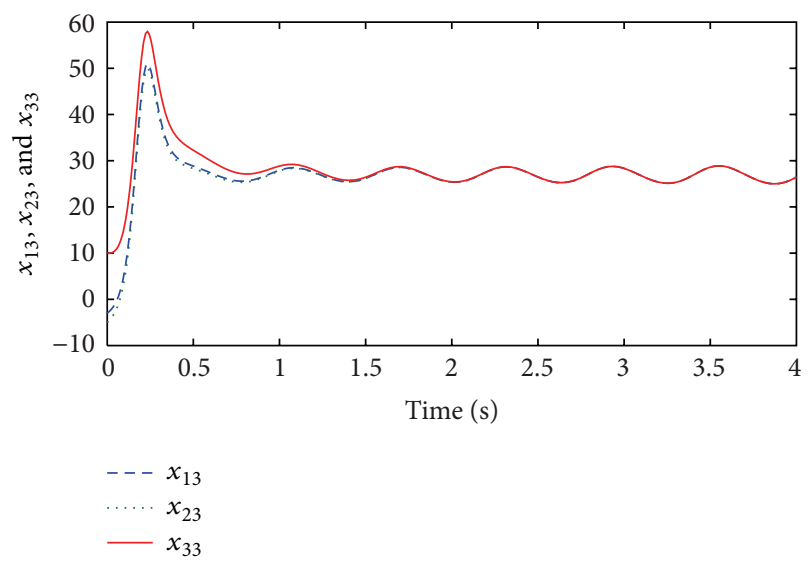

FIgURE 4: The state trajectories $x_{13}, x_{23}$, and $x_{33}$ of the Lorenz systems with time $t$.

where

$$
\begin{aligned}
& A_{1}=\left[\begin{array}{ccc}
-35 & 35 & 0 \\
-7 & 28 & 0 \\
0 & 0 & -3
\end{array}\right], \quad A_{2}=\left[\begin{array}{ccc}
-36 & 36 & 0 \\
0 & 20 & 0 \\
0 & 0 & -3
\end{array}\right], \\
& A_{3}=\left[\begin{array}{ccc}
-10 & 10 & 0 \\
28 & -1 & 0 \\
0 & 0 & -\frac{8}{3}
\end{array}\right] \text {, } \\
& g_{1}\left(x_{1}\right)=\left[\begin{array}{c}
0 \\
-x_{11} x_{13} \\
x_{11} x_{12}
\end{array}\right], \quad g_{2}\left(x_{2}\right)=\left[\begin{array}{c}
0 \\
-x_{21} x_{23} \\
x_{21} x_{22}
\end{array}\right] \text {, } \\
& g_{3}\left(x_{3}\right)=\left[\begin{array}{c}
0 \\
-x_{31} x_{33} \\
x_{31} x_{32}
\end{array}\right]
\end{aligned}
$$

and $D_{1}=\operatorname{diag}\left(d_{11}, d_{12}, d_{13}\right), D_{2}=\operatorname{diag}\left(d_{21}, d_{22}, d_{23}\right)$, and $D_{3}=\operatorname{diag}\left(d_{31}, d_{32}, d_{33}\right)$ are the coupled matrices, and $u_{1}=$ $\left[u_{11}, u_{12}, u_{13}\right]^{T}$ and $u_{2}=\left[u_{21}, u_{22}, u_{23}\right]^{T}$ are the control inputs.
Let the synchronization error state be $\dot{e}_{i-1}=\dot{x}_{i}-\dot{x}_{i-1}$, $(i=2,3)$. The error dynamical equations are

$$
\begin{aligned}
\dot{e}= & {\left[\begin{array}{ll}
\Delta_{1} & \Delta_{2} \\
\Delta_{3} & \Delta_{4}
\end{array}\right] e } \\
& +\left[\begin{array}{c}
-x_{11}-x_{12}+u_{11} \\
7 x_{11}-8 x_{12}-x_{21} x_{23}+x_{11} x_{13}+u_{12} \\
x_{21} x_{22}-x_{11} x_{12}+u_{13} \\
26 x_{21}+26 x_{22}+u_{21}-u_{11} \\
28 x_{21}-21 x_{22}-x_{31} x_{33}+x_{21} x_{23}+u_{22}-u_{12} \\
\frac{1}{3} x_{23}+x_{31} x_{32}-x_{21} x_{22}+u_{23}-u_{13}
\end{array}\right],
\end{aligned}
$$

where

$$
\begin{aligned}
& \Delta_{1}=\left[\begin{array}{ccc}
-36-d_{11}-d_{21} & 36 & 0 \\
0 & 20-d_{12}-d_{22} & 0 \\
0 & 0 & -3-d_{13}-d_{23}
\end{array}\right], \\
& \Delta_{2}=\left[\begin{array}{ccc}
-d_{11} & 0 & 0 \\
0 & -d_{12} & 0 \\
0 & 0 & -d_{13}
\end{array}\right], \quad \Delta_{3}=\left[\begin{array}{ccc}
d_{21} & 0 & 0 \\
0 & d_{22} & 0 \\
0 & 0 & d_{23}
\end{array}\right], \\
& \Delta_{4}=\left[\begin{array}{ccc}
-10-d_{31} & 10 & 0 \\
28 & -1-d_{32} & 0 \\
0 & 0 & -\frac{8}{3}-d_{33}
\end{array}\right] .
\end{aligned}
$$

The controllers $u_{1}$ and $u_{2}$ are designed as

$$
\begin{gathered}
u_{1}=v_{1}-\left(A_{2}-A_{1}\right) x_{1}-g_{2}\left(x_{2}\right)+g_{1}\left(x_{1}\right), \\
u_{2}=v_{2}+v_{1}-\left(A_{3}-A_{1}\right) x_{1}-g_{3}\left(x_{3}\right)+g_{1}\left(x_{1}\right),
\end{gathered}
$$

where

$$
v_{1}=\left[\begin{array}{cccccc}
0 & 0 & 0 & 0 & 0 & 0 \\
-36 & 0 & 0 & 0 & 0 & 0 \\
0 & 0 & 0 & 0 & 0 & 0
\end{array}\right] e
$$$$
v_{2}=\left[\begin{array}{cccccc}
d_{11}-d_{21} & 0 & 0 & 0 & 0 & 0 \\
36 & d_{12}-d_{22} & 0 & -38 & 0 & 0 \\
0 & 0 & d_{13}-d_{23} & 0 & 0 & 0
\end{array}\right] e .
$$

The error systems (24) become

$$
\dot{e}=\left[\begin{array}{ll}
\Delta_{1}^{*} & \Delta_{2}^{*} \\
\Delta_{3}^{*} & \Delta_{4}^{*}
\end{array}\right] e
$$

where

$$
\begin{aligned}
& \Delta_{1}^{*}=\left[\begin{array}{ccc}
-36-d_{11}-d_{21} & 36 & 0 \\
-36 & 20-d_{12}-d_{22} & 0 \\
0 & 0 & -3-d_{13}-d_{23}
\end{array}\right], \\
& \Delta_{2}^{*}=\left[\begin{array}{ccc}
-d_{11} & 0 & 0 \\
0 & -d_{12} & 0 \\
0 & 0 & -d_{13}
\end{array}\right], \quad \Delta_{3}^{*}=\left[\begin{array}{ccc}
d_{11} & 0 & 0 \\
0 & d_{12} & 0 \\
0 & 0 & d_{13}
\end{array}\right], \\
& \Delta_{4}^{*}=\left[\begin{array}{ccc}
-10-d_{31} & 10 & 0 \\
-10 & -1-d_{32} & 0 \\
0 & 0 & -\frac{8}{3}-d_{33}
\end{array}\right] .
\end{aligned}
$$




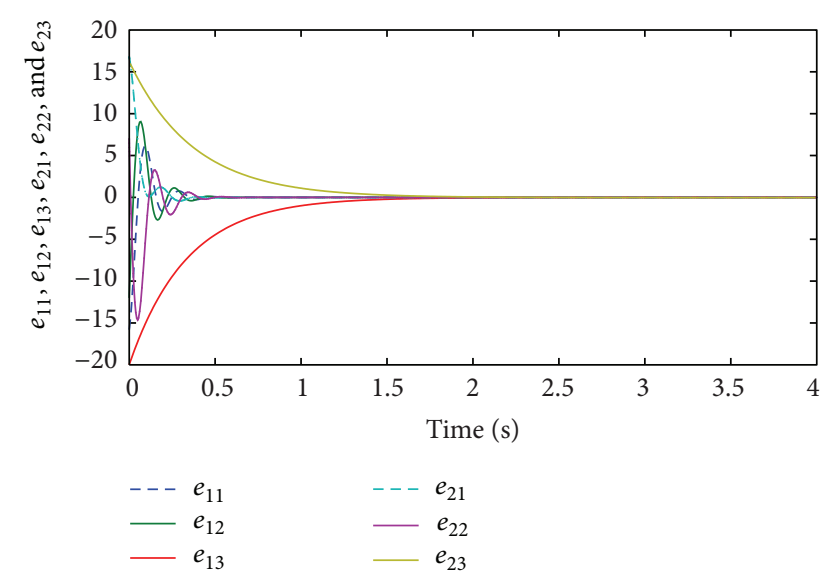

Figure 5: Dynamics of the variables $e_{11}, e_{12}$, and $e_{13}$ of the error system $e_{1}$ with time $t$.

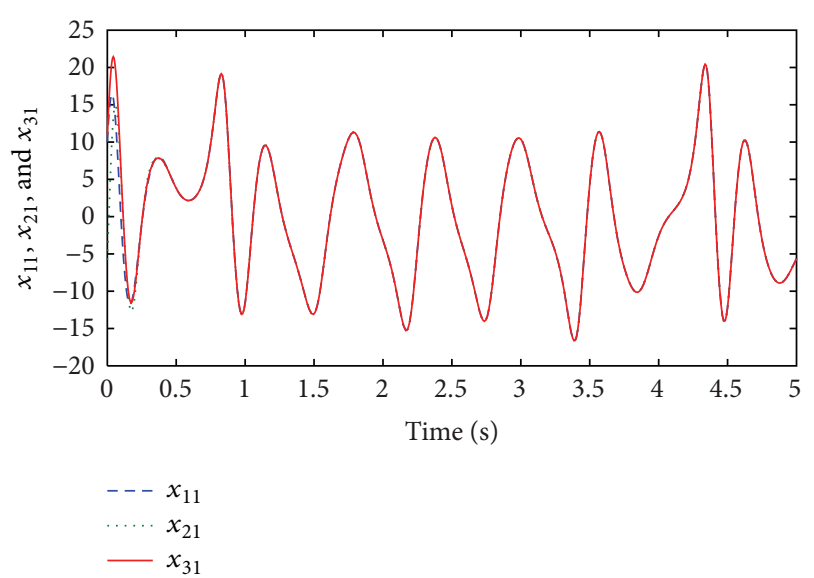

FIGURE 6: The state trajectories $x_{11}, x_{21}$, and $x_{31}$ of the chaotic systems with time $t$.

From Theorem 1, we know that the conditions

$$
\begin{aligned}
& -36-d_{11}-d_{21}<0, \quad 20-d_{12}-d_{22}<0, \\
& -3-d_{13}-d_{23}<0, \quad-10-d_{31}<0, \\
& -1-d_{32}<0, \quad-\frac{8}{3}-d_{33}<0
\end{aligned}
$$

ensure that the error systems (28) are asymptotically stable at the origin under the controllers $u_{1}$ and $u_{2}$. Thus, the synchronization between the response systems and the drive systems is realized.

Similar to Case 1, let the initial conditions of the drive system and the response systems be $\left(x_{11}(0), x_{12}(0), x_{13}(0)\right)=$ $(10,20,30),\left(x_{21}(0), x_{22}(0), x_{23}(0)\right)=(-5.8,8,10)$, and $\left(x_{31}(0), x_{32}(0), x_{33}(0)\right)=(11,15,26)$, respectively. And we choose that $d_{11}=d_{21}=d_{13}=d_{23}=d_{31}=d_{33}=0$, $d_{12}=10, d_{22}=11$, and $d_{32}=1$. The state trajectories of the error systems and chaotic systems are shown in Figures $5,6,7$, and 8 , respectively. It is easy to see that the state variables and the error variables all tend towards to less than $1.5 \mathrm{~s}$ under the controllers. Simulation results demonstrate

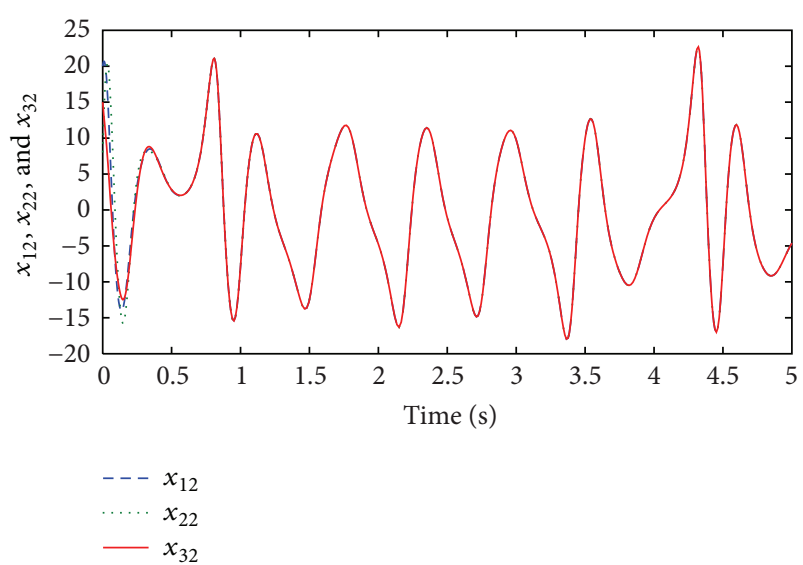

FIGURE 7: The state trajectories $x_{12}, x_{22}$, and $x_{32}$ of the chaotic systems with time $t$.

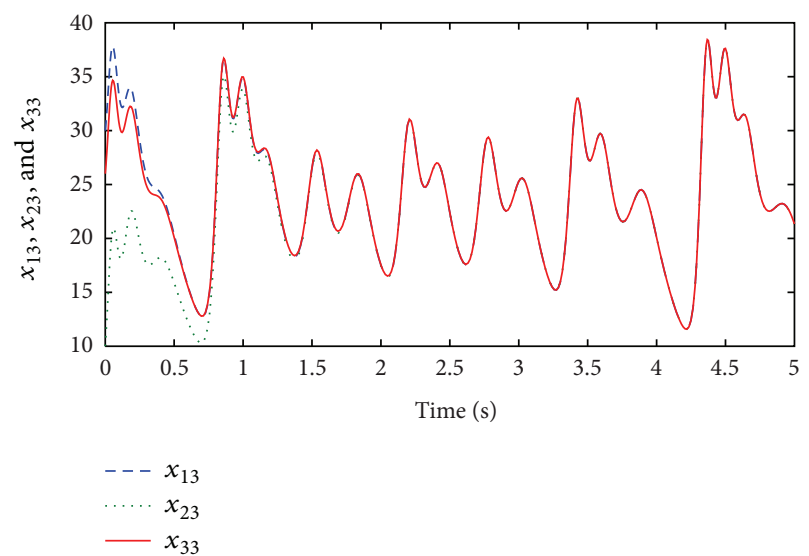

FIgURE 8: The state trajectories $x_{13}, x_{23}$, and $x_{33}$ of the chaotic systems with time $t$.

that the convergence rates are much faster than the earlier research results proposed in the literature [19, 21]; then the effectiveness of the synchronization control schemes is confirmed.

\section{Conclusions}

This paper concerns the synchronization of $N$ coupled chaotic systems with ring connection. A direct design control method is firstly used to design the controllers. The synchronization of $N$ coupled chaotic systems is realized by coupling the state variables. This technology will undoubtedly improve performance of secret signaling and possess better application value in practice. Furthermore, our presented strategy can ensure strict synchronization between $N$ coupled chaotic systems. This will lead to a rapid development in multilateral communications.

\section{Acknowledgments}

The author's work was supported in part by the Applied Mathematics Enhancement Program (AMEP) of Linyi University 
and the National Natural Science Foundation of China, under Grants 61273012 and 61273218 , by a project supported by the Scientific Research Fund of Zhejiang Provincial Education Department under Grant Y201223649, by a Project of Shandong Province Higher Educational Science and Technology Program under Grants J13LI11 and J12LI58.

\section{References}

[1] L. M. Pecora and T. L. Carroll, "Synchronization in chaotic systems," Physical Review Letters, vol. 64, no. 8, pp. 821-824, 1990.

[2] B. Nana, P. Woafo, and S. Domngang, "Chaotic synchronization with experimental application to secure communications," Communications in Nonlinear Science and Numerical Simulation, vol. 14, no. 5, pp. 2266-2276, 2009.

[3] C. Aguirre, D. Campos, P. Pascual, and E. Serrano, "Synchronization effects using a piecewise linear map-based spiking-bursting neuron model," Neurocomputing, vol. 69, no. 10-12, pp. 11161119, 2006.

[4] S. Tang and J. M. Liu, "Chaos synchronization in semiconductor lasers with optoelectronic feedback," IEEE Journal of Quantum Electronics, vol. 39, no. 6, pp. 708-715, 2003.

[5] J.-J. Yan, Y.-S. Yang, T.-Y. Chiang, and C.-Y. Chen, "Robust synchronization of unified chaotic systems via sliding mode control," Chaos, Solitons and Fractals, vol. 34, no. 3, pp. 947-954, 2007.

[6] S. Guan, C.-H. Lai, and G. W. Wei, "Phase synchronization between two essentially different chaotic systems," Physical Review E, vol. 72, no. 1, Article ID 016205, 8 pages, 2005.

[7] N. Cai, Y. W. Jing, and S. Y. Zhang, "Adaptive synchronization and anti-synchronization of two different chaotic systems," Acta Physica Sinica, vol. 58, no. 2, pp. 802-813, 2009.

[8] N. Cai, Y. Jing, and S. Zhang, "Generalized projective synchronization of different chaotic systems based on antisymmetric structure," Chaos, Solitons and Fractals, vol. 42, no. 2, pp. 11901196, 2009.

[9] J. Cao, D. W. C. Ho, and Y. Yang, "Projective synchronization of a class of delayed chaotic systems via impulsive control," Physics Letters A, vol. 373, no. 35, pp. 3128-3133, 2009.

[10] W. He and J. Cao, "Generalized synchronization of chaotic systems: an auxiliary system approach via matrix measure," Chaos, vol. 19, no. 1, Article ID 013118, 10 pages, 2009.

[11] Q. Gan, R. Xu, and P. Yang, "Synchronization of non-identical chaotic delayed fuzzy cellular neural networks based on sliding mode control," Communications in Nonlinear Science and Numerical Simulation, vol. 17, no. 1, pp. 433-443, 2012.

[12] J. Lu, J. Cao, and D. W. C. Ho, "Adaptive stabilization and synchronization for chaotic Lur'e systems with time-varying delay," IEEE Transactions on Circuits and Systems I, vol. 55, no. 5, pp. 1347-1356, 2008.

[13] W. Yu, G. Chen, J. Lü, and J. Kurths, "Synchronization via pinning control on general complex networks," SIAM Journal on Control and Optimization, vol. 51, no. 2, pp. 1395-1416, 2013.

[14] Q. Gan and Y. Liang, "Synchronization of chaotic neural networks with time delay in the leakage term and parametric uncertainties based on sampled-data control," Journal of the Franklin Institute, vol. 349, no. 6, pp. 1955-1971, 2012.

[15] Q.-L. Zhang, "Synchronization of multi-chaotic systems via ring impulsive control," Journal of Control Theory and Applications, vol. 27, no. 2, pp. 226-232, 2010.
[16] Y. Yu and S. Zhang, "Global synchronization of three coupled chaotic systems with ring connection," Chaos, Solitons and Fractals, vol. 24, no. 5, pp. 1233-1242, 2005.

[17] J.-A. Lu, X.-P. Han, Y.-T. Li, and M.-H. Yu, "Adaptive coupled synchronization among multi-Lorenz systems family," Chaos, Solitons and Fractals, vol. 31, no. 4, pp. 866-878, 2007.

[18] G. Grassi, "Propagation of projective synchronization in a series connection of chaotic systems," Journal of the Franklin Institute, vol. 347, no. 2, pp. 438-451, 2010.

[19] Y. Liu and L. Lü, "Synchronization of $N$ different coupled chaotic systems with ring and chain connections," Applied Mathematics and Mechanics, vol. 29, no. 10, pp. 1299-1308, 2008.

[20] Y. Tang and J.-A. Fang, "Synchronization of N-coupled fractional-order chaotic systems with ring connection," Communications in Nonlinear Science and Numerical Simulation, vol. 15, no. 2, pp. 401-412, 2010.

[21] X. Chen and J. Qiu, "Synchronization of $N$ different chaotic systems based on antisymmetric structure," Mathematical Problems in Engineering, vol. 2013, Article ID 742765, 6 pages, 2013.

[22] L.-X. Yang and J. Zhang, "Synchronization of three identical systems and its application for secure communication with noise perturbation," in Proceedings of the International Conference on Information Engineering and Computer Science (ICIECS '09), pp. 1-4, Wuhan, China, December 2009. 


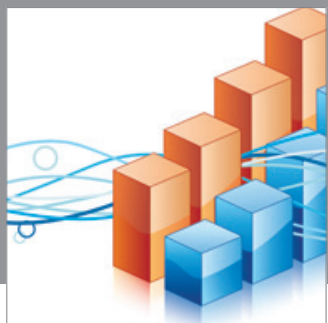

Advances in

Operations Research

mansans

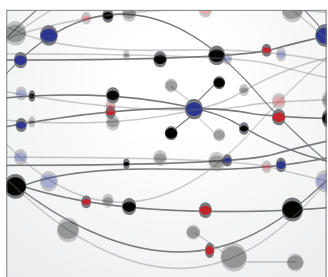

The Scientific World Journal
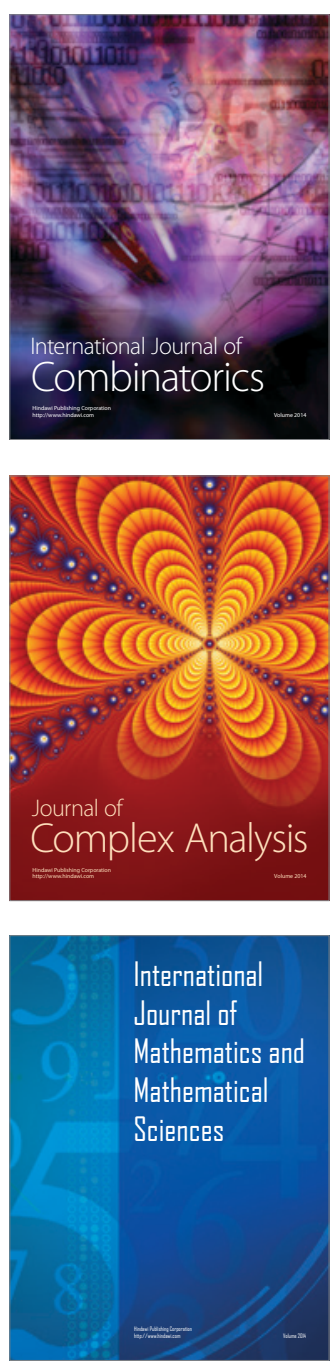
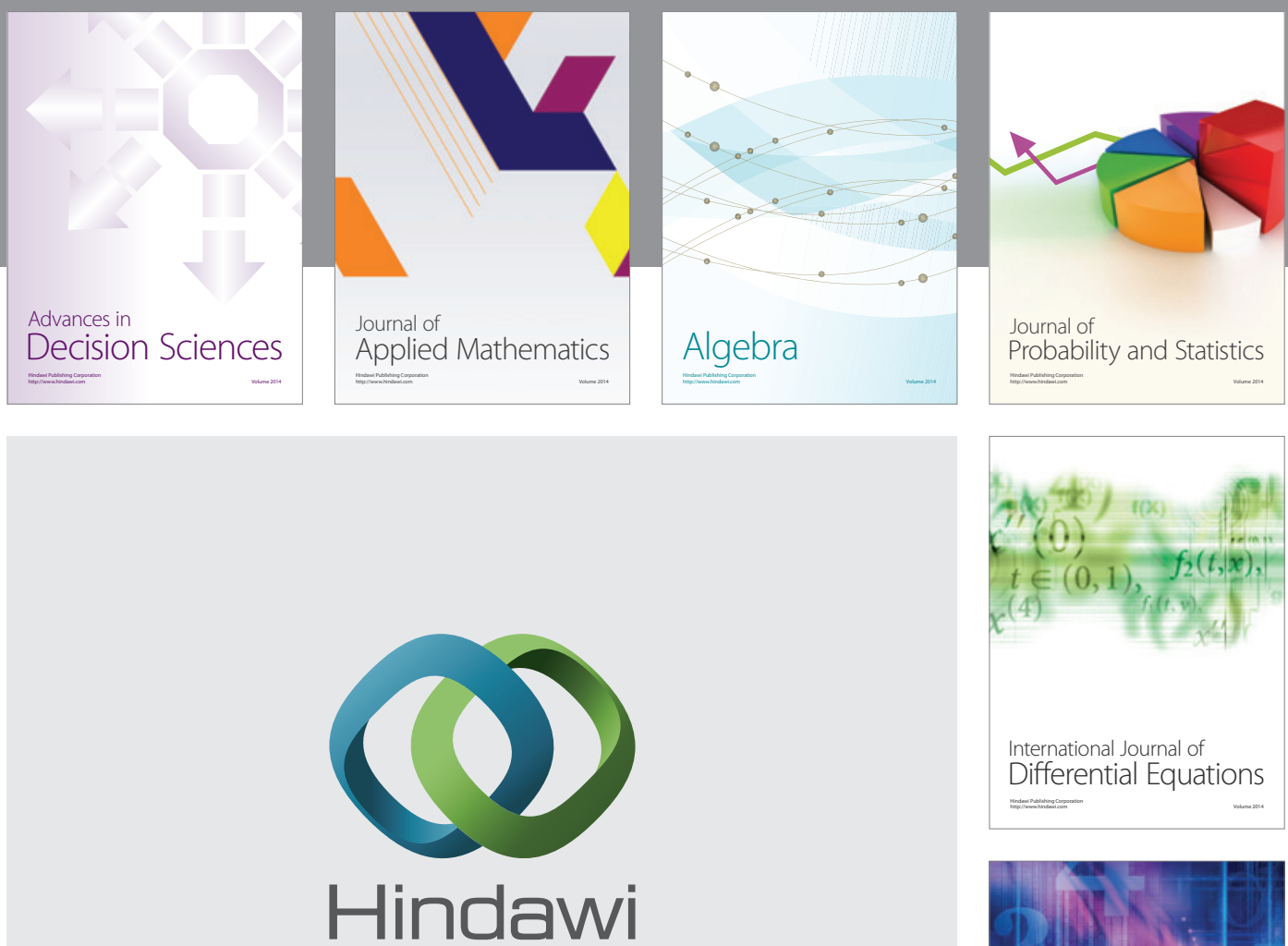

Submit your manuscripts at http://www.hindawi.com
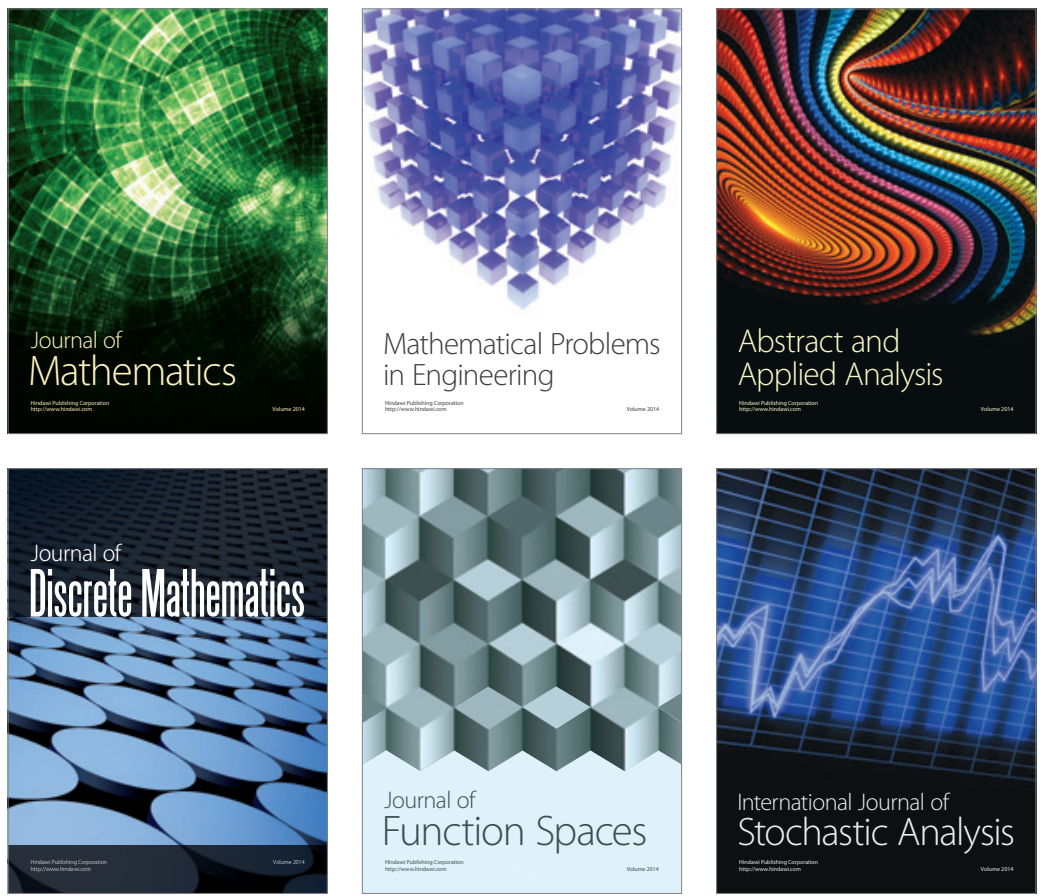

Journal of

Function Spaces

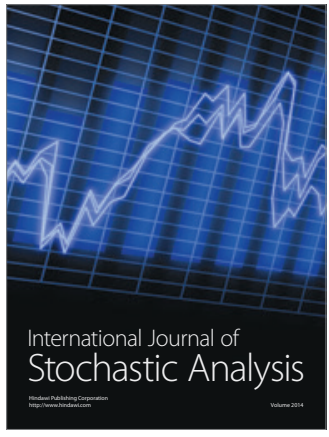

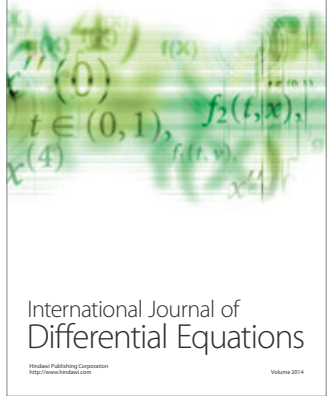
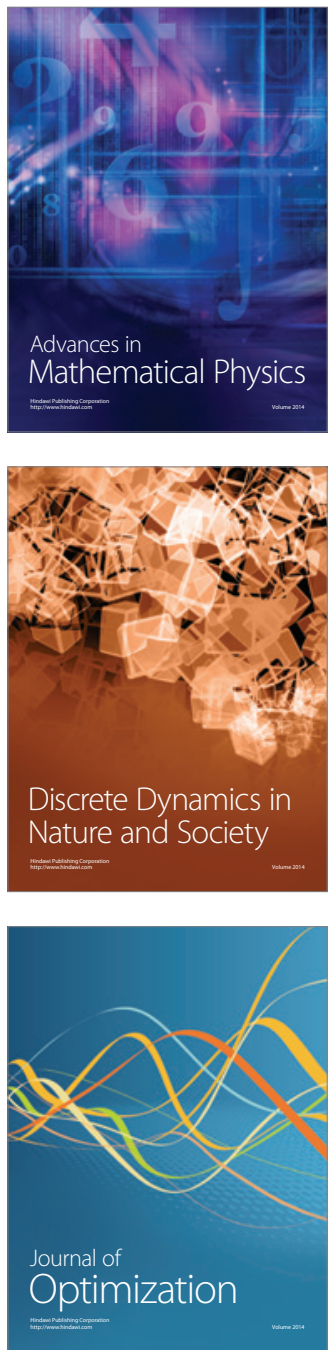